\title{
Molecular Validation of Putative Antimicrobial Peptides for Improved Human Immunodeficiency Virus Diagnostics via HIV Protein p24
}

Monray Edward Williams ${ }^{1}$, Marius Tincho, Musa Gabere ${ }^{2}$, Ashley Uys $^{3}$, Mervin Meyer ${ }^{1}$ and Ashley Pretorius ${ }^{1 *}$

${ }^{1}$ University of the Western Cape, South Africa

${ }^{2}$ King Abdullah International Medical Research Center, National Guard Health Affairs, Saudi Arabia

${ }^{3}$ Medical Diagnostech PTY LTD, South Africa

\begin{abstract}
Objective: Various HIV diagnostic systems are available however; the p24 antigen detection assay is preferred due to its ability to decrease the window period for HIV detection. The p24 antigen assay has been associated with low sensitivity. Antimicrobial Peptides (AMPs) display huge potential in development of more effective diagnostics tools. The aim of this study was to design derivative AMPs from AMPs identified previously, using an in silico approach, as templates, with higher predicted binding affinity for the p24 protein and implement the best suited AMPs in a Point-OfCare (POC) device for detection of HIV-1 and HIV-2.
\end{abstract}

Method: This study firstly used an in silico approach to identify derivative AMPs which bind the N-terminal domain of the p24 antigen with increased predicted binding affinity. The in silico approach used the parental AMPs as templates for generation of the derivative AMPs through site-directed mutagenesis, 3-D structure prediction and docking studies. Secondly, the binding between the synthetically synthesized AMPs and the HIV antigen p24 was validated by an in house Lateral Flow Device (LFD) "on/off" binding experiment. The most promising AMPs were conjugated to gold nanoparticles (AuNPs) and implemented in a LFD to detect HIV-1 and HIV-2 using patient sera as well as a panel of international HIV standards (panel C10).

Result: With conjugation of AMPs to gold nanoparticles, parent AMP 1 and derivative AMP 1.1 used in combination most sensitively detected the recombinant protein p24 and was therefore selected for development of a LFD prototype for HIV diagnostics. Global HIV-1 (C10) and HIV-2 (C10) standards were successfully detected by the LFD prototype as well as HIV infected serum samples.

Conclusion: A sensitive HIV-1 and HIV-2 LFD prototype was developed which would within 15 minutes provide patients with accurate and sensitive diagnosis of HIV.

Keywords: HIV pandemic; Early HIV diagnostics; p24 antigen; Antimicrobial Peptides; in silico approach; Protein-peptide interactions; Nanotechnology; Lateral flow device

\section{Introduction}

The Human Immunodeficiency Virus-1 (HIV-1) was first identified in 1983 and to date it has progressed to obtain pandemic status. HIV is a slow progressing lentivirus that causes Acquired Immunodeficiency Syndrome (AIDS) [1]. HIV functions by attacking the T-helper cells which could include dendritic cells, macrophages and $\mathrm{CD} 4^{+}$cells. All these cells play imperative roles in the establishment of the immune effector responses such as the cytotoxic T-lymphocyte (CTL) and $\beta$-cell responses [2,3]. T-helper cells are thus arguably the most important cells within the adaptive immunity as they are required for almost all the adaptive immune responses [2-4]. They function by activating $\beta$-cells to secrete antibodies and macrophages to destroy ingested microbes. Additionally they also activate the cytotoxic T-cells to kill infected target cells [2-5]. AIDS patients suffer from a lack in the number of T-helper cells, consequently suffering from a weakened immune system, which promotes secondary infections such as Tuberculosis [1].

As of the year 2013, it was estimated that more than 36.9 million people, globally were diseased with HIV, and these numbers are increasing daily [6]. According to literature, it appears that the less developed countries experience a greater extent of the disease burden. Statistics designates Sub-Saharan Africa (SSA) as the worlds most affected region, with an estimate of 22.9 million people living with AIDS. This accounts for $60 \%$ of the world's HIV population. Swaziland has the world's largest prevalence rate $(25.9 \%)$ whereas South Africa is known to have the world largest HIV infected population (5.6 million)
[7]. This statistics are attributed to the poor current treatment regiments and the ineffective diagnostic systems.

Current HIV diagnostics is based on two methods of detection. It either relies on the detection of antibodies that the patient produced in an attempt to resist the virus or detection of the whole virus or particles of the virus. The main goal of such diagnostic systems is to enforce early detection of the virus and subsequently initiating treatment. This, in turn would enable patients to develop accommodating lifestyles.

One of the most common utilized diagnostic systems is the p24 antigen assay. The p24 antigen detection test (NEN HIV-1 p24 EIA assay) measures the amount of free p24 in a serological sample, extracted two weeks after infection, via Enzyme Immuno Assay (EIA) [8]. This method is an improvement when compared to antibody specific tests, since it is able to decrease the window period by a few

*Corresponding author: Ashley Pretorius, Department of Biotechnology, University of the Western Cape, Life Science Building (Room 2.48), Bellville, South Africa, Tel: + 27 (0) 21959 9727; +27 (0) 82329 5646; Fax: + 27 (0) 21959 3505; E-mail: aspretorius@uwc.ac.za

Received March 14, 2016; Accepted April 26, 2016; Published May 03, 2016

Citation: Williams ME, Tincho M, Gabere M, Uys A, Meyer M, et al. (2016) Molecular Validation of Putative Antimicrobial Peptides for Improved Human Immunodeficiency Virus Diagnostics via HIV Protein p24. J AIDS Clin Res 7: 571 doi:10.4172/2155-6113.1000571

Copyright: (c) 2016 Williams ME, et al. This is an open-access article distributed under the terms of the Creative Commons Attribution License, which permits unrestricted use, distribution, and reproduction in any medium, provided the original author and source are credited. 
days by successful detection of the p24 protein. Accordingly providing early diagnosis, which translates into immediate treatment of an infected individual [8-10].

However, the p24 antigen assay is associated with an insensitive detection limit and seldom used as a sole indicator of an HIV positive individual. This insensitivity may be explained by the presence of the anti-p24 antibody within sera samples. In many instances after infection, the p24 antibody produced by the host, binds to the C-terminal of the p24 protein [8-11]. This interaction obstructs the detection of the p24 protein by the p24 test, since it requires an unoccupied C-terminal domain. Hence as much as $50 \%$ of asymptomatic HIV infected individuals display a HIV negative result. This concern may be circumvented by using a molecule (e.g. peptide) to target an additional pocket on the p24 protein or, by simply breaking the interaction of the p24 antibody and the p24 protein prior to conducting the assay, which will allow for detection of the p24 protein by the current diagnostic methods [12].

Work done by Sutthent et al. reported on an improved p24 antigen diagnostic test, however it requires a "booster" step in which serum and plasma samples require pre-heating before testing [12]. This preheating step breaks the complex formed between the p24 protein and circulating antibodies and allows detection of the p24 protein. However, the introduction of a heating step makes the detection more labor demanding and may decrease the efficacy of the test. Additionally it cannot be a called a Point-of-Care test as it requires numerous steps to complete (non-rapid), thus making it difficult to implement in resource limited areas. Therefore, the aim of this study to employ a molecule (e.g. a peptide) targeting a specific binding site on the 24 protein, which would potentially translate into improved diagnostics of HIV.

Antimicrobial Peptides (AMPs) display huge potential in this regard. AMPs are a subset of proteins that forms part of the innate immune response. They serve as the primary defense line in many organisms, including both prokaryotes and eukaryotes [13-15]. They are widely distributed in plants, invertebrates and vertebrates where they exhibit a broad spectrum of activity against pathogens. These include antifungal, antiprotozoal, antibacterial as well as antiviral activity [15]. It is highly unlikely that pathogens can develop resistance against AMPs due to their diversity, broad spectra of activity and unique mode of action [16,17]. AMPs target the bacterial membrane, therefore, if a microbe were to develop resistance against AMPs, it would be required to redesign its membrane and change its lipid composition. Since most microbial species prefer expending the least amount of energy to maintain survival, 'developing' AMP resistance is considered to be an energy costly solution [17].

In a previous study, novel AMPs were identified using an in silico mathematical algorithm HMMER (Hidden Markov Model) [18] (unpublished data). The HMMER algorithm was used to create various predictive models from different super-families (Fish and Crabs, Amphibians, Insects, Human Defensins, Plants, Vertebrates and Microorganisms), which was used to scan various databases. This resulted in an output of 30 putative AMP sequences with potential antiHIV activity. These 30 AMPs were ranked according to their e-value, with the lowest e-value predicted to have the highest anti-HIV activity. The top ten AMP's 3-D structures were determined using I-TASSER and docked against the HIV proteins gp120 and p24 using Patchdock. Results from this study showed that several of the AMPs bind p24 at the N-terminal domain, whilst several AMPs bind gp120 at the V1/ V2 domain [unpublished data]. The binding of several AMPs to the $\mathrm{N}$-terminal domain of p 24 would potentially address the shortcomings of the current p24 assay [8-12]. A preliminary in vitro study conducted at Medical Diagnostech PTY LTD $\left(\mathrm{MD}^{\circ}\right)$, using the ten top ranked AMPs based on their e-values, showed that two of these AMPs, 1 and 8, recorded the highest G-score when used to detect HIV 1 and 2 in serum samples. Based on the algorithm used to generate the putative AMPs, these AMPs were predicted to potentially have anti-HIV activity. The anti-HIV activity is achieved through binding of these AMPs to HIV specific proteins. Through this binding to specific HIV proteins, the AMPs can thus similarly be used as a diagnostic approach.

Thus the aims of the study was: (i) To use the parental AMPs as templates for the identification of derivative AMPs, which bind the p24 $\mathrm{N}$-terminal domain with greater affinity, demonstrated through in silico binding studies, (ii) To molecularly validate the binding between the AMPs and the 24 protein using a "on/off" binding experiment in a LFD setting and (iii) To develop a prototype with these specific AMPs conjugated to gold nanoparticles (AuNPs) to accurately and sensitively detect the HI virus within patient samples

\section{Materials and Methods}

\section{In silico study}

In silico site-directed mutagenesis: The aim of the in silico approach was to use the two parental AMPs as templates to generate derivative AMPs that display increased predicted binding affinity for the N-terminal domain of the p24 protein. Amino acids of AMPs play imperative roles in its functioning, hence, creating AMP derivatives by site-directed mutagenesis are often beneficial [19]. Before the commencement of in silico site-directed mutagenesis, essential amino acids have to be identified. Knowledge-based FADE and Contacts (KFC) server (http://kfc.mitchell-lab.org/upload.php) was used to identify "hotspot" residues or mutation sensitive residues to effectively perform in silico site-directed mutagenesis. The KFC server is based on two predictive models namely; basis of shape specificity features and the other based on biochemical contacts [20]. It is stated that KFC displays better predictive accuracy than computational alanine scanning (Robetta-Ala) for determining mutation sensitive residues [21]. The structural complexes between the parent AMPs (determined from a previous study) and HIV protein p24 were uploaded to the online software and the results were displayed as all residues within the binding interface and those residues within the interface that is considered as "hotspots". All amino acids substituted to generate derivate AMPs where of similar characteristics to the amino acids present in the parental AMPs, as to maintain the predicted activity and functioning of the AMPs.

Physicochemical properties: All derivative AMP sequences were submitted to the AMP characterization software Bactibase (physiochemical properties tab) (http://bactibase.pfba-lab- tun.org/ physicochem) [14] and Antimicrobial Peptide Database (prediction tab) (http://aps.unmc.edu/AP/prediction/prediction_main.php) [22] to determine the characteristics of each derivative AMP.

In silico 3-D structure prediction: Derivative AMPs (1.1 and 8.1) 3-D structures were predicted using the online software I- TASSER (http://zhanglab.ccmb.med.umich.edu/I-TASSER). I-TASSER is a 3-D structure prediction tool that makes use of a de-novo (or ab-initio) approach. I-TASSER predicts structures of a protein purely from its primary amino acid sequence using principles of physics that govern protein folding and/or make use of information derived from known structures but without relying on any evolutionary relationship to known folds. The amino acid sequences of derivative AMPs and the 
p-chain of the HIV protein p24 (PDB I.D 1E6J) were used as an input for the software [23]. Predicted structure outputs were given as PDB files, with a scoring which represented the accuracy of each structure prediction.

\section{In silico protein-protein interaction study}

The online protein-protein interaction server PatchDock server was used in this study based on its advantages over the other available docking tools. PatchDock uses a very efficient algorithm for proteinsmall ligand and protein-protein docking, which is based on a rigidbody geometric hashing algorithm. PatchDock is also one of the only servers that score the binding affinity. PatchDock is aimed at finding docking transformations that yield good molecular shape complementarity. The PatchDock algorithm divides the Connolly dot surface representation of the molecules into concave, convex and flat patches. Thereafter complementary patches are matched in order to generate candidate transformations. Each candidate transformation is further evaluated by a scoring function that considers both geometric fit and atomic desolvation [24]. A geometric score is provided where a higher score is indicitive of better binding.

Each derivative AMP 3-D structure was docked against the HIV protein p24 3-D structure respectively. The binding affinities were recorded and the results were downloaded as PDB files, which represented the structural complex between the mutated AMPs and the HIV protein p24. Following the result output from PatchDock, visualization analysis was done by PyMol (OS) software. Emphasis was placed on the binding affinity score and binding pocket utilized by the respective derivative AMPs.

\section{Molecular study}

Peptide synthesis and protein expression: Parental (AMP 1 and AMP 8) and derivative (AMP 1.1 and AMP 8.1) antimicrobial peptides were selected for chemical synthesis (sequences not provided PCT application Patent P1600GB00). The aforementioned AMPs were synthesized at a purity of $>98 \%$ and purchased from GL Biochem (Shanghai).

The p24 DNA sequence (GenScript, USA) was cloned into a pGEX6P-2 vector between the Bam $\mathrm{H} 1$ and NotI sites. The provided vector was transformed into E.coli Bl21 cells and purified using the GST purification system. All samples were analyzed by a $12 \%$ SDS-PAGE. The purified $\mathrm{p} 24$ protein was lyophilized and stored at $-80^{\circ} \mathrm{C}$ for further use in binding study.

Binding validation: In house LFD binding assay: In this study we have developed an "on/off" protein-peptide interaction protocol where protein and AMP are allowed to interact in a LFD set-up. This method allows for easy and quick determination of which AMP combination best bind the recombinant HIV protein p24. The intensity of detection bands represented in the test window of the LFD would be an indication of which AMP pair best detect or bind the p24 antigen. Results from this experiment were visually interpreted by the Dot-Blot intensity using an in-house G1-G10 colour coded chart (Medical Diagnostech PTY LTD, South Africa (MD ${ }^{\circ}$ ) (Figure 1). A Negative result is indicated by the absence of a dot whereas a positive result is indicated by the presence of a red dot in the test window.

Preparation of AuNPs-AMP conjugates: Stable colloidal AMPs conjugates were prepared according to an "in house" protocol designed by Medical Diagnostech $\left(\mathrm{MD}^{\circ}\right)$. Various AMP combinations were tested. Biotinylated AMPs were used in the conjugate preparation

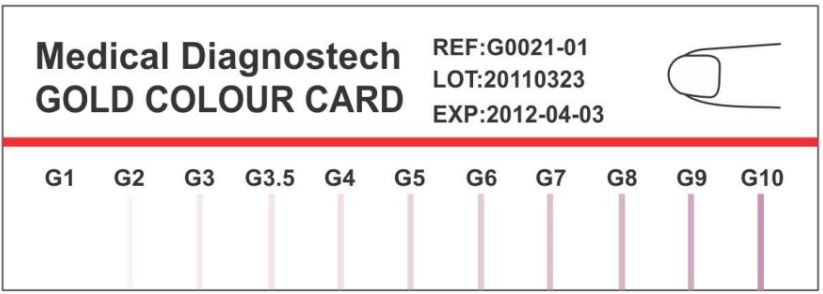

Figure 1: G-scale Gold Colour Card for interpretation of binding intensity.

whereas non-biotinylated AMPs were used on the membrane of the LFD. A biotinylation kit from Thermo Scientific (EZ-Link NHS-PEG4Biotinylation kit) was used to biotinylate AMPs used in conjugates. Biotinylated AMPs and streptavidin stocks were made up to $0.05 \mathrm{mg} /$ $\mathrm{ml}$. The biotinylated AMPs were added to $10 \mu \mathrm{g}$ of streptavidin in the ratio 1:1, 1:2,1:3, and 1:4. This was allowed to complex for 30 minutes. This mixture was conjugated to $2 \mu \mathrm{g}$ gold nanoparticles and allowed to incubate for 15 minutes. Thereafter $500 \mu \mathrm{l}$ of $10 \%$ BSA was added and incubated for a further 15 minutes. The conjugated mixture was then centrifuged for 30 minutes at $3300 \mathrm{rpm}$. The supernatant was discarded and the pellet resuspended in $50 \mu \mathrm{l}$ of borate suspension buffer. To each of the resuspended conjugates, $1 \%$ casein, $8 \%$ sucrose, $0.75 \%$ Tween 20 , and $0.01 \%$ glycerol were added. The conjugate was then blotted at $2 \mu \mathrm{l}$ per test and run as per test instructions.

Preparation of attachment of AMPs to membrane: The AMPs that had to be attached to the membrane were diluted with membrane buffer containing $25 \mathrm{mM}$ Borate $\mathrm{pH}$ 8.0. This membrane buffer was further optimized to contain ethanol and surfactants Triton-X 100 and Tween.

Assembly of Strips: Strips were assembled in accordance with the current "in-house" HIV system created by Medical Diagnostech (MD $\left.{ }^{\circledR}\right)$. AMPs were blotted on the nitrocellulose membranes in triplicate at a concentration of $1 \mathrm{mg} / \mathrm{ml}$. The successfully produced AMP conjugates were formulated and blotted onto the conjugate pads of the strips. Different combinations of the AMPs were experimented upon to determine the optimal AMP pair for p24/HIV detection. The strips were then placed into cassettes and the test was performed.

Testing of recombinant antigen p24 sample: Recombinant p24 protein samples were serially diluted, and $10 \mu \mathrm{g} / \mathrm{ml}$ was applied with $120 \mu \mathrm{L}$ of HIV running buffer to the sample well and results were read after 15 minutes. After 30 minutes results were not considered.

Testing of HIV-1 and HIV-2 samples: Negative and HIV-1 positive blood and serum were sourced from the Western Province Blood Transfusion Services (South Africa). HIV-2 positive sera were sourced from NIBSC and Fapon. For the respective testing, $10 \mu \mathrm{l}$ the patient samples as well as HIV standards (C10) were applied to the sample well of the LFD. Furthermore $120 \mu \mathrm{L}$ of HIV running buffer was added to the sample well and results were read at 15 minutes. After 30 minutes results were not considered.

\section{Results}

\section{In silico study}

Physcochemical properties: Overall physicochemical properties remained fairly similar following substitution based site-directed mutagenesis with a slight change in one or two parameters (results shown in Table 1). AMP 1 had no change in its overall net charge whereas AMP 
Citation: Williams ME, Tincho M, Gabere M, Uys A, Meyer M, et al. (2016) Molecular Validation of Putative Antimicrobial Peptides for Improved Human Immunodeficiency Virus Diagnostics via HIV Protein p24. J AIDS Clin Res 7: 571. doi:10.4172/2155-6113.1000571

Page 4 of 7

8.1 had an increase in its net positive charge by +1 . AMP 1 showed a slight change from a complete extended structure to an extended-partial $\alpha$-helix structure (AMP 1.1) and AMP 8.1 retained its $\beta$-hairpin structure (Figures 2 A-D). With these slight changes in physicochemical characteristics post site-directed mutagenesis, the derivative AMPs still contain physiochemical features associated with this class of peptides (Table 1).

Both derivative AMPs displayed an increased in binding affinity to the 24 protein (Table 2). AMP 1.1 had an increase in binding affinity from 14708 to 15328 and AMP 8.1 displayed an increase from 9418 to 10704. Binding of the derivative AMPs remained at the $\mathrm{N}$-terminal domain with slight shifting in their binding position (Figure 3). AMP 8 initially bound at a position between the $\mathrm{N}$-terminal and C-terminal domain, however AMP 8.1 experienced a noticeable shift in binding, which resulted in its binding at the $\mathrm{N}$-terminal domain (Figure 4). The change in the 3-D structure, caused by the introduction of the mutation, displayed a better-predicted fit at binding pockets at the N-terminal domain when parental AMPs and derivative AMPs were compared.

\section{Molecular study}

Recombinant protein expression: Successful protein expression was

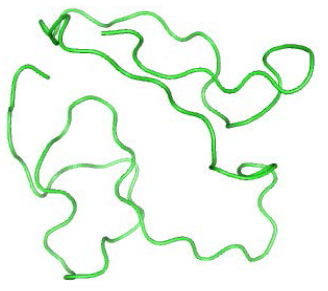

(A)

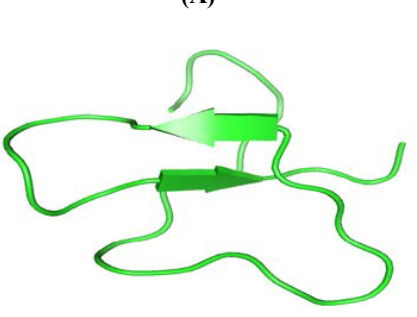

(C)

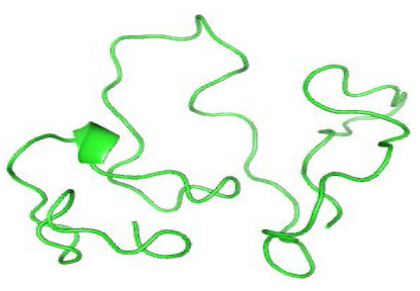

(B)

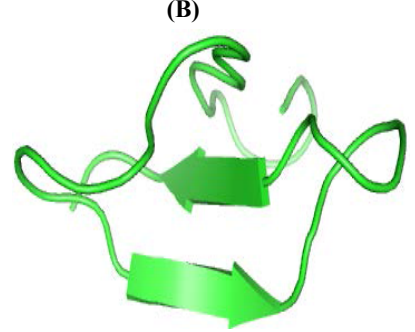

(D)
Figure 2: The predicted 3-D structure of the parental and mutated AMPs. (A) Parent AMP 1 and (B) the derivative AMP 1.1, (C) Parent AMP 8 and (D) the derivative AMP 8.1. achieved, with successful cleavage of the GST-p24 fusion protein. A $12 \%$ SDS PAGE display the resolved fusion protein $(\sim 49 \mathrm{kDa})$, and the cleaved p24 protein $(\sim 24 \mathrm{kDa})$ as compared to the size marker (Figure 5). This was expected since the predicted size of the $\mathrm{p} 24$ protein is $24 \mathrm{kDa}$. The purified p24 protein sample was stored at $-80^{\circ} \mathrm{C}$ until use in the binding assay.

Parent AMP 1 and derivative AMP 1.1 used in combination displayed the greatest binding to the purified p24 protein as indicated by the G-score within the LFD (Table 3). As shown by Figure 6, a signal response corresponding to the test line on the LFD was observed when testing the positive 24 protein sample diluted in serum, in comparison to the p24 negative sample (serum only).

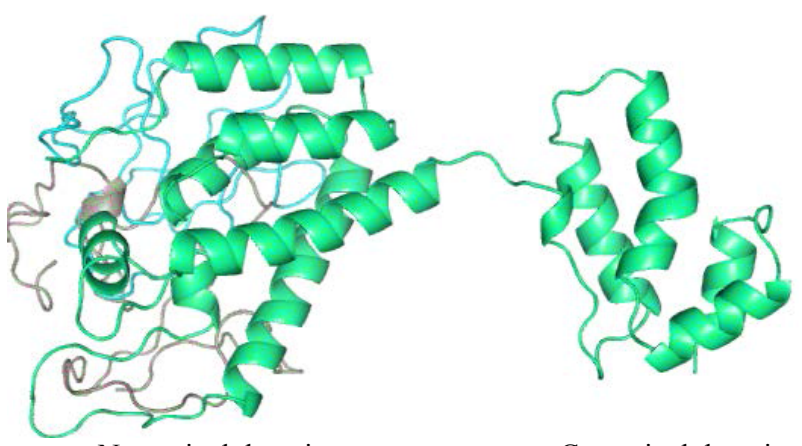

N-terminal domain

C-terminal domain

Figure 3: Displays the binding shift of the parent AMP 1 (turquoise) to the derivative AMP 1.1 (grey) on the p24 protein (green).

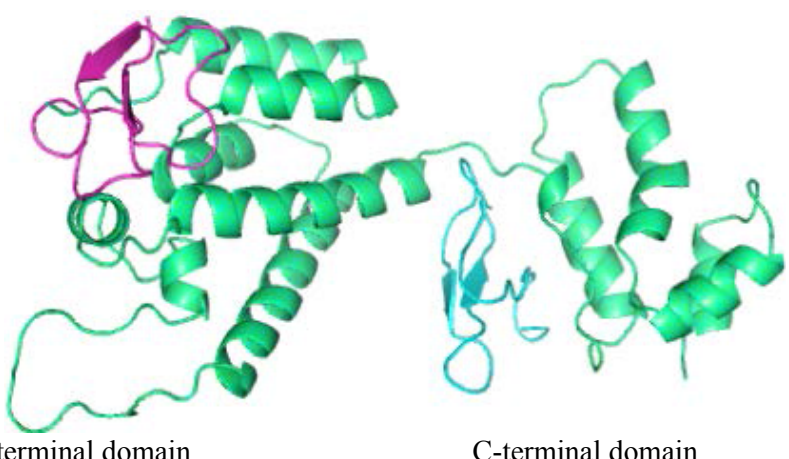

$\mathrm{N}$-terminal domain

C-terminal domain

Figure 4: Displays the binding shift of the parent AMP 8 (turquoise) to the derivative AMP 8.1 (purple) on the p24 protein (green).

\begin{tabular}{|c|c|c|c|c|c|c|c|c|c|c|c|c|}
\hline AMP & Arg \% & Lys \% & Cys \% & Hydrophobicity & $\begin{array}{l}\text { Molecular } \\
\text { weight }\end{array}$ & $\begin{array}{l}\text { Total Net } \\
\text { charge }\end{array}$ & Size & pl & $\begin{array}{c}\text { Boman } \\
\text { index }\end{array}$ & $\begin{array}{l}\text { Instability } \\
\text { index }\end{array}$ & $\begin{array}{l}\text { Half-life: } \\
\text { mammal }\end{array}$ & $\begin{array}{c}\text { Common Amino } \\
\text { Acids }\end{array}$ \\
\hline AMP 1 & 6 & 11 & 16 & 34 & 8903.716 & +6 & 79 & 8.3 & 2.17 & 43.71 & $1.2 \mathrm{~h}$ & Cys \\
\hline AMP 1.1 & 6 & 11 & 16 & 34 & 8942.752 & +6 & 79 & 8.3 & 2.18 & 44.30 & $1.2 \mathrm{~h}$ & Cys \\
\hline AMP 8 & 5 & 14 & 17 & 38 & 3670.552 & +8 & 34 & 9.6 & 1.07 & 48.28 & $1.2 \mathrm{~h}$ & Cys \\
\hline AMP 8.1 & 5 & 14 & 17 & 35 & 3660.516 & +9 & 34 & 9.6 & 1.29 & 48.28 & $1.2 \mathrm{~h}$ & Cys \\
\hline
\end{tabular}

Table 1: Physiochemical properties of parent and derivative AMPs determined using Bactibase and Antimicrobial Peptide Database.

\begin{tabular}{|l|c|c|c|}
\hline Molecule & Binding affinity of parent AMPs & Binding pocket of parent AMPs & $\begin{array}{c}\text { Difference in Increase } \\
\text { Binding affinity }\end{array}$ \\
\hline AMP 1 & 14708 & N-terminal & - \\
\hline AMP 1.1 $\left(\mathrm{F}_{62} \mathrm{~W}\right)$ & 15328 & N-terminal & 620 \\
\hline AMP 8 & 9418 & Between N and C-terminal domain & $4.2 \%$ \\
\hline AMP 8.1 $\left(\mathrm{F}_{12} \mathrm{H}\right)$ & 10704 & N-terminal & - \\
\hline
\end{tabular}

Table 2: Predicted Binding affinities, position of binding to HIV protein p24 and percentage differences in binding affinity. The Binding affinity is a geometric scoring of the interaction between p24 and AMP. It does not have binding unit to the scoring system. In silico docking. 


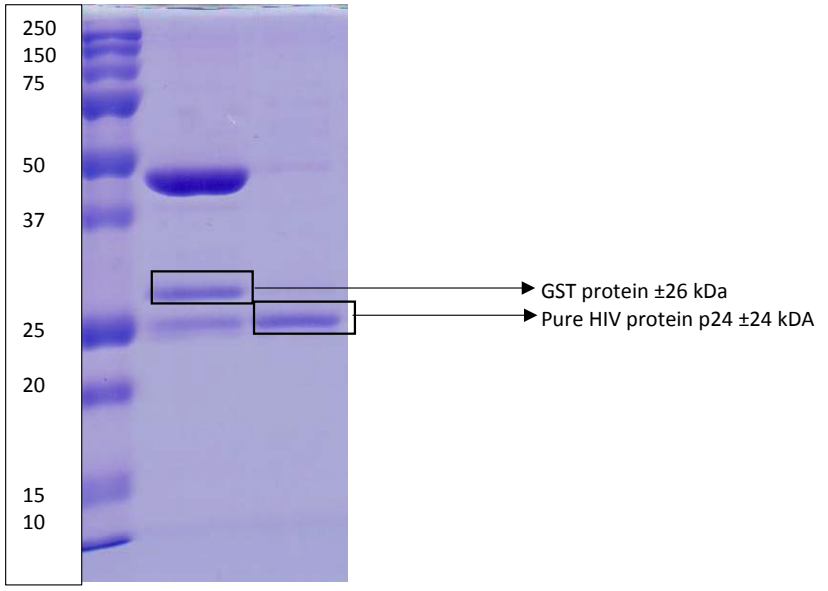

Figure 5: SDS-PAGE analysis of pure purified HIV protein p24 after cleavage by protease HRV 3C. Lane 1: $250 \mathrm{kDa}$ presission plus marker, lane 2: pooled purified GST-tagged p24 protein and lane 3: cleaved pure protein p24.

\begin{tabular}{|c|c|c|}
\hline AMP & Sample tested & G rating \\
\hline AMP 1 & $\mathrm{p} 24$ & $\mathrm{G} 8$ \\
\hline AMP 1.1 & $\mathrm{p} 24$ & $\mathrm{G} 10$ \\
\hline AMP 8 & $\mathrm{p} 24$ & $\mathrm{G} 4$ \\
\hline AMP 8.1 & $\mathrm{p} 24$ & $\mathrm{G} 6$ \\
\hline
\end{tabular}

Table 3: G-score ratings of various AMPs binding the p24 antigen using a LFD binding assay. In house protein-protein LFD binding study.

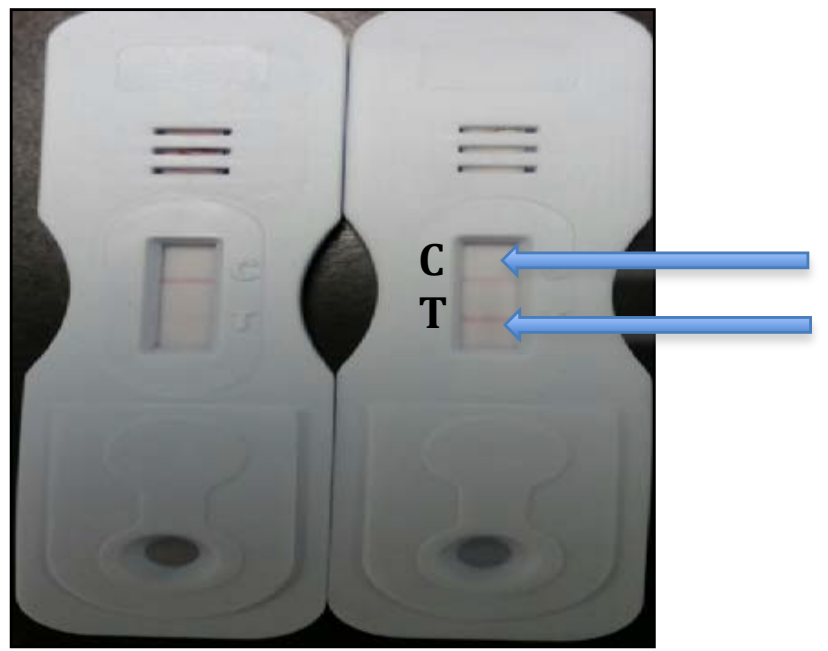

(A)

(B)

Figure 6: LFD binding assay using AMP 1 and AMP 1.1 to detect the recombinant p24 protein. AMP 1 was used on the conjugate pad and AMP 1.1 was used on the membrane of the LFD, as this AMP combination most effectively detected the recombinant p24 protein. The LFD tested a (A) p24 negative sample, (B) recombinant protein p24.

Prototype development: The combination of Parent AMP 1 (conjugate) and derivative AMP 1.1 (membrane) displayed the greatest G-score for the $\mathrm{p} 24$ protein within the LFD, therefore it was predicted that this AMP combination would also detect HIV positive samples when implemented in a LFD (Figure 7).

Positive standards HIV-1 (C10) and HIV-2 (C10) were tested on the

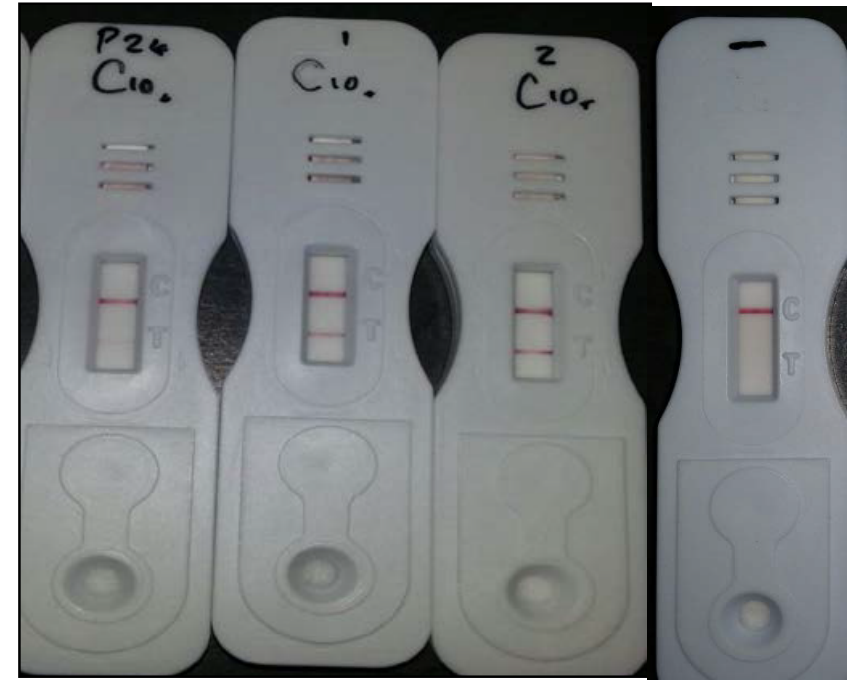

(A)

(B)

(C)

(D)

Figure 7: The AMP 1/AMP 1.1 in a LFD prototype for HIV detection. This AMP combination best detected the p24 antigen therefore used in combination to test the (A) Recombinant protein p24, (B) HIV-1 standard (C) HIV-2 standard and $(D)$ negative control.

AMP specific prototype at Medical Diagnostech Laboratories. Results indicate that the AMP prototype displayed no signal intensity for the HIV negative samples, slight intensity for the p24 antigen sample and great band intensities for the HIV-1 and HIV-2 samples.

\section{Discussion}

Various HIV diagnostic systems are currently implemented in an attempt to diagnose the virus. However many suffer various shortcomings. The main goal of this diagnostic system is to enforce early detection of the virus and subsequently assist medical practitioners towards the correct treatment plan. This, in turn would enable patients to develop accommodating lifestyles. AMPs have shown great promise in circumventing the drawbacks associated with the current diagnostic systems, when implemented in LFDs. Antibodies have been the gold standard for detection of samples in LFDs, due to its sensitivity of detection of targets within samples. However, they have recently been linked to various shortcomings, such as cross-reactivity and sequence coverage. This is evident in the current HIV p24 antigen detection assay, where detection antibodies have overlapping epitopes to natural host antibodies, which often result in false negatives obtained during testing. Other shortcomings include a labour intensive antibody preparation, time consummation, limited assay utility, and nonspecific binding to non-target molecules [25]. Peptides are immediate solutions due to numerous properties that overcome the shortcomings associated with antibodies. These include its small size; therefore rapid and reproducible synthesis, simple and controllable modification, high stability and non/low toxicity. In addition as seen in this study, peptides may be fine-tuned to perform certain functions, which make them a favorable choice in diagnostic development [26].

In a previous study, novel AMPs (AMP 1 and AMP 8) were identified using an in silico mathematical algorithm HMMER (Hidden Markov model). In silico protein-protein interactions studies displayed that the identified AMPs were able to bind to a terminal domain of the HIV p24 protein that could be used for improved HIV diagnostics. Based on these results, these AMPs were considered candidates for possible 
diagnostic markers for use in a Point-Of-Care device as they bind to a different epitope to that of the p24 antibody, which is used in the current p24 diagnostic test. The first aim of the research was to use the parental AMPs as templates to develop derivative AMPs that displays increased binding affinity for the $\mathrm{N}$-terminal domain of the $\mathrm{p} 24$ protein using in silico site-directed mutagenesis. Thereafter to molecularly validate it's binding to the $\mathrm{p} 24$ protein and finally to implement the best suited AMPs in a LFD to detect HIV within samples.

Through in silico studies completed by this study, data suggests after in silico site-directed mutagenesis, all derivative AMPs displayed an increased in binding affinity to the $\mathrm{p} 24$ protein by at least $4.2 \%$. Binding of the derivative AMPs remained at the N-terminal domain (Table 3) with slight shifting in their binding position (Figure 3). AMP 8 initially bound at a position between the N-terminal and C-terminal domain, however AMP 8.1 experienced a shift in binding, which resulted in its binding at the $\mathrm{N}$-terminal domain (Figure 4). The $\mathrm{N}$-terminal domain presents a larger surface area for binding of AMPs, thus with the incorporation of proper mutation to AMP 8 , shifting in binding pocket to the N-terminal domain was achieved. This increase in binding affinity and utilization of N-terminal domain provides an opportunity for the development of a LFD for HIV detection.

Before testing these AMPs within a LFD, a binding validation had to be performed between the HIV protein p24 and respective AMPs. This study made use of an "in house" LFD (MD) to validate AMPs that displayed the best binding affinity for the HIV protein $\mathrm{p} 24$. Various combinations of the four AMPs were used in the development of the LFD, to test which AMP combination most effectively detected/bound the recombinant p24 protein. In this study we made use of the HIV-1 p24 recombinant protein (designated RH24, derived from HIV-1 strain HXB2, group $M$, subtype $B$ ). Group $M$ is responsible for the majority of infections globally including Africa. Within group $M$, the average inter-subtype genetic variability is $15 \%$ for the gag (p24) gene. Previous studies indicate regardless of inter-subtype genetic variability the currently used serological and molecular diagnostic tests for HIV-1 are still sufficiently specific and sensitive to detect the most prevalent HIV1 genetic forms worldwide, as also indicated in this study [27].

Parent AMP 1 and derivative AMP 1.1 displayed the greatest G-score for the p24 protein within the LFD. As illustrated in Figure 6B, a response was experienced when testing the $\mathrm{p} 24$ positive protein, in comparison to the p24 negative sample (Figure 6A). In addition AMP 1 and derivative AMP 1.1 was also predicted to have to greatest antiHIV properties (previous study) and displayed the greatest predicted binding affinity for the p24 protein according to the in silico research. Therefore, the in silico approaches has effectively predicted binding affinity of AMPs to the $\mathrm{p} 24$ protein. Based on the results presented, AMP 1 and AMP 1.1 were thus best suited to be implemented in a LFD prototype for HIV detection.

The prototype strongly detects the presence of HIV-1 and HIV-2 global standards (Figure 7B and Figure 7C), and to a lesser degree the p24 antigen (Figure 7A). HIV-1 and HIV-2 share many similarities including their basic gene arrangement, modes of transmission, intracellular replication pathways, clinical consequence and more importantly both result in AIDS [28]. Therefore it is expected that their detection should be of similar intensity. In this LFD a concentration of $10 \mu \mathrm{g} / \mathrm{ml}$ of recombinant protein was applied to the LFD. Previous studies $[28,29]$ indicate that HIV positive patient samples contain a mean concentration of $200 \mathrm{pg} / \mathrm{ml}$ of the p24 antigen. Therefore the sample concentration applied to the LFD prototype would then be extremely concentrated and may be experiencing what is known as the "hook effect".
The "hook effect" occurs when too much antigen is added to a LFD and results in false negatives or lower signal intensities. When extremely concentrated samples are applied to the LFD, the sample would migrate to the conjugate pad where it would recognize the AuNP conjugated AMPs. However, due to the sample being highly concentrated, the AuNP-AMP conjugate would become saturated with the antigen at a very quick rate and the excess HIV positive antigens would already migrate to the test line. Here the non-labeled conjugates would bind to the capture AMPs and which would allow for less or no gold conjugates to bind at the capture line, subsequently lower or no signal intensity. One way to overcome this is to either dilute samples before application, or increase the concentration of AuNPs-AMP conjugates in the conjugate pad to enable capturing all HIV positive antigens [14, 30,31]

\section{Conclusion}

The LFD prototype accurately detected both HIV-1 and HIV-2, providing reproducible results on HIV-1 standards, HIV-2 standards, as well as patient samples. Preliminary data suggest that this LFD is able to out compete current commercially available HIV diagnostic devices on basis of sensitivity, accuracy, lower sample requirement, and result interpretation within 15 minutes of the test being taken.

\section{Future work}

This study provides many avenues of furthering the research capacity, which essentially would enable further optimization of these LFD prototypes. One area of research would be to determine the kinetics of binding between the AMPs and p24 protein by the use of a biophysical technique such as Surface Plasmon resonance (SPR). These results could be compared to current LFD binding assay results presented in this study. In addition SPR could be used to perform a comparative study by determining the kinetics of binding between the identified AMPs and various HIV proteins to identify which proteins the AMPs preferentially bind. Another avenue of research would be to elucidate the structural binding between respective AMPs and p24 antigen by Nuclear Magnetic Resonance. This would be a good comparative study between the in silico and molecular structural interactions demonstrated by this research. These prototypes will be optimized by attempting to remove the "hook effect" to ensure accurate results. A field study will be completed to test $100+$ patient samples at various stages on infection to assess the consistency and detection power of the prototype.

\section{Acknowledgements}

The authors of this study would like to acknowledge all funding contributors, the National Research Foundation, Medical Research Council, Department of Science and technology and the MINTEK/ Nanotechnology Innovation Centre. Also special thanks to Medical Diagnostech for their contribution to the research project.

\section{Funding Contributors}

National Research Foundation, Department of Science and Technology/ Mintek, Nanotechnology innovation centre and the Medical Research Council

\section{References}

1. Quaranta M, Mattioli G, Vella S (2012) Review Article: Glances in Immunology of HIV and HCV Infection. Advances in Virology 1: 1-13.

2. Chinen J, Shearer W (2002) Molecular Virology and Immunology of HIV Infection. Allergy and Clinical Immunology 1: 189-198.

3. Roy S, Wodarz D (2012) Infection of HIV-specific CD4 T helper cells and the clonal composition of the response. Journal of Theoretical Biology 304: 143151.

4. Fanales-Belasio E, Raimondo M, Suligoi B, Buttò S (2010) HIV virology and pathogenetic mechanisms of infection: a brief overview. HIV virus and pathogenicity 46 (1): 5-14. 
Citation: Williams ME, Tincho M, Gabere M, Uys A, Meyer M, et al. (2016) Molecular Validation of Putative Antimicrobial Peptides for Improved Human Immunodeficiency Virus Diagnostics via HIV Protein p24. J AIDS Clin Res 7: 571. doi:10.4172/2155-6113.1000571

5. Alberts B, Johnson A, Lewis J, Raff M, Roberts K, et al. (2002) Molecular Biology of the Cell. (4th edn), Garland Science, New York.

6. Glynn M, Kinahan D, Durcee J (2013) CD4 counting technologies for HIV theraphy monitoring in resource-poor settings-state-of-the-art and emerging microtechnologist. Lab Chip 13: 2731-2748.

7. Feldblum PJ, Latka M, Lombaard J, Chetty C, Chen PL, et al. (2012) HIV incidence and prevalence amoun cohorts of women with higher risk behaviour in Bloemfontein and Rustenburg, South Africa: a prospective study. BMJ Open 2: $1-8$.

8. Iweala O (2004) HIV diagnostic tests: An overview. Contraception 70: 141-147.

9. Buttò S, Suligoi B, Fanales-Belasio E, Raimondo M (2010) Laboratory diagnostics for HIV infection. Laboratory Diagnostics for HIV infection 46: 24 33.

10. Cornett J, Kirn T (2013) Laboratory Diagnosis of HIV in Adults: A Review of Current Methods. Medical Microbiology 1: 1-7.

11. Monaco-Malbet S, Berthet-Colominas C, Novelli A, Battaı N, Piga N, et al. (2000) Mutual Conformational Adaptations in Antigen and Antibody upon Complex Formation between an Fab and HIV-1 Capsid Protein p24. Structure 8: 1069-1077.

12. Sutthent R, Gaudart N, Chokpaibulkit K, Tanilang N, Kanoksinsombath C, et al. (2003) p24 antigen detection assay modified with a booster step for diagnosis and mentoring of human immunodeficiency virus type 1 infection. Clinical Microbiology 1: 1016-1022.

13. Brogden K (2005) Antimicrobial Peptides: Pore formers or Metabolic inhibitors on bacteria. Nature reviews 1: 1-13.

14. Wong R, Tse H (2009) Lateral Flow Immunoassay. (1st edn), Humana Press, New York.

15. Giuliani A, Pirri G, Nicolleto S (2007) Antimicrobial peptides: an overview of a promising class of therapeutics. Central European Journal of Biology 1: 1-33.

16. Yeaman M, Yount N (2003) Mechanism of antimicrobial peptide action and resistance. Pharmacological Reviews 55: 27-55

17. Zasloff M (2002) Antimicrobial peptides of multicellular organisms. Nature 1: 389-395.

18. Finn R, Clements J, Eddy S (2011) HMMER web server: interactive sequence similarity searching . Nucleic Acids Research 1: 29-37.
19. Biro J (2006) Amino acid size, charge, hydropathy indices and matrices for protein structure analysis. Theoretical Biology and Medical Modelling 1: 1-12.

20. Darnell S, LeGault L, Mitchell J (2008) KFC server: interactive forecasting of protein interaction hotspots. Nucleic Acids Research 36: 265-269.

21. Hammami R, Zohir A, Chrisophe LeLay JH, Fliss I (2010) BACTIBASE second release: a database and tool platform for bacteriocin characterization. BMC Microbiology 10: 1-5

22. Wang Z, Wang G (2004) APD: the Antimicrobial Peptide Database. Nucleic Acids Research 32: 590-592.

23. Zhang Y (2008) I-TASSER server for protein 3D structure prediction. Biomed Central 40: 1-8.

24. Schneidman-Duhovny D, Inbar Y, Nussinov R, Wolfson H (2005) PatchDock and SymmDock: servers for rigid and symmetric docking. Nucleic Acids Research 1: 1-5.

25. Siddiqui M (2010) Monoclonal antibodies as Diagnostics; An Appriasal. Indian Journal of Pharmaceutical Sciences 1: 12-17.

26. Carmona S, Sartor P, Leguizamon M, Campetella O, Aguero F (2012) Diagnostic Peptide Dsicovery, Prioritization of pathogen diagnostic markers using multiple Features. PloS one 7: 1-14.

27. Bunaguro L, Tornesello M, Bunaguro F (2007) Human immunodeficiency Virus Type 1 Subtype Distribution in the World Wide Epidemic: Pathogenic and Therapeutic implication . Journal of virology 81 (18): 10209-10219.

28. Nyamweya S, Hegedus A Jaye A Sarah Rowland-Jones KFDM (2013) Comparing HIV-1 and HIV-2 infection: Lessons for viral immunonopathogenesis. Reviews in Medical Virology 23: 221-240.

29. Elluarie M, Rubistein A (1991) Correlation of serum antigen and antibody concentration with clinical features in HIV infection. Archives of Disease in Childhood 66: 200-203.

30. Lourens A, Jarvis J, Meintjies G, Samuel C (2014) Rapid Diagnostics of Cryptococcol Meningitis by Use of Lateral Flow Assay on Cerebral Spinal Fluid Samples: Influence of he High Dose 'Hook' Effect. Journal of Clinical Microbiology 52: 4172-4175.

31. Luchavez J, Baker J, Alcantara S, Belizario V, Cheng Q, et al (2011) Laboratory Demonstration of Prozone-like Effect in HRP2-detecting Malaria Rapid Diagnostic Tests: Implications for Clincal Management. Malaria Journal 10: 1-7. 